\title{
L'expression du multiple par le corps de la peinture
}

\author{
Quote: PAQUET, Bernard. L'expression du multiple par le corps de la peinture. \\ Porto Arte: Revista de Artes Visuais. Porto Alegre: PPGAV-UFRGS, v. 23, n. 38, \\ p.1-7, jan.-jun. 2018. e-ISSN 2179-8001. Dol: http://dx.doi.org/10.22456/2179- \\ 8001.80770
}

Résumé: Cet essai présente une expérimentation poétique et de réflexion qui construit une image humaine du futur (posthumain) sur la base de l'anatomie sans le sexe ni la peau. Pour multiplier ce corps, en peinture, deux paramètres sont pris en considération: les éléments anatomiques qui se prêtent facilement à de multiples variations et puis l'absence de la dualité.

Mots-clés: Cyborg. Posthumain. Peinture. Multiple.

Depuis quelques années, je travaille en peinture sur une représentation imaginaire de l'humain du futur que je qualifie de posthumain. Cette figure est inspirée de la médecine régénérative qui avance que le corps pourra dans un proche avenir assurer sa longévité en se régénérant par la culture de ses propres tissus ou de tissus artificiels. II se distinguerait à cet effet des corps qui seraient réparés exclusivement à l'aide de prothèses mécaniques et électroniques. Dans cette optique, je conçois et construis des corps sans épiderme composés de pièces anatomiques colorées en exploitant une liberté d'expression au regard de la réalité morphologique humaine. Je les place au centre d'univers qui paraissent être d'étranges paradis selon les modes figuratif, symbolique et métaphorique, sur des toiles de grandes dimensions. J'y conjugue, par une séparation rappelant l'expression de l'horizon, un réalisme de type illusionniste avec des à-plats dorés afin d'introduire des incertitudes temporelles et spatiales (Figures 1, 3 et 5).

Dans ces univers, les corps ne sont ni des robots, ni des cyborgs bourrés de composantes électroniques ; ils se projettent plus loin dans le futur, étant avant toute chose des constructions d'éléments anatomiques libres. Outre cela, ils forment des organismes sans genre qui ignorent le féminin et le masculin. Une partie de cette production est formée d'œuvres de plus petits formats qui affichent des fragments anatomiques multicolores, inventés pour composer une sorte de banque anatomique ouverte et ludique. Cette vision picturale représente le corps humain du futur comme une structure souple et colorée qui sera régénérée en permanence par la multiplication d'éléments variables, autonomes, permutables et amovibles.

\section{LE STATUT DU CORPS}

Pendant plusieurs siècles en Occident, le corps fut considéré comme un tout irréductible et sacré dont on connaissait peu le fonctionnement interne, car il était une créature de Dieu. À la Renaissance, avec l'idée que l'homme se plaçait dorénavant au centre du monde, les premières explorations importantes furent réalisées par le belge Vésale (1514-1564) qui effectua des dissections de cadavres pour mettre à jour l'anatomie et ainsi en faire un objet de découvertes, tout comme son contemporain le français Charles Estienne ou l'espagnol Juan Valverde de Amusco. Le corps fut par conséquent graduellement désacralisé et conçu comme un ensemble de composantes ${ }^{1}$ de moins en moins mystérieuses mais toujours reliées à la finalité de la Nature et du Divin. Dans les siècles suivants, ces composantes seront au contraire traitées de manière plus fonctionnelle, mécanique et scientifique alors qu'il était évident que l'organisme humain pouvait dès lors se décomposer en fragments qu'il était possible d'isoler et étudier. C'est au XVIIème siècle le cas de Diderot avec son encyclopédie, de Descartes avec ses corps-machines ou encore de la collection du Musée de La Specola à Florence exposant des corps et des fragments entièrement fabriqués de cire. Le siècle suivant verra de nombreuses publications qui seront souvent le fruit de collaborations entre médecins et artistes comme par exemple l'ouvrage de J M Bourgery et $\mathrm{N} \mathrm{H}$ Jacob: Traité complet de l'anatomie de l'homme 1830-1850.

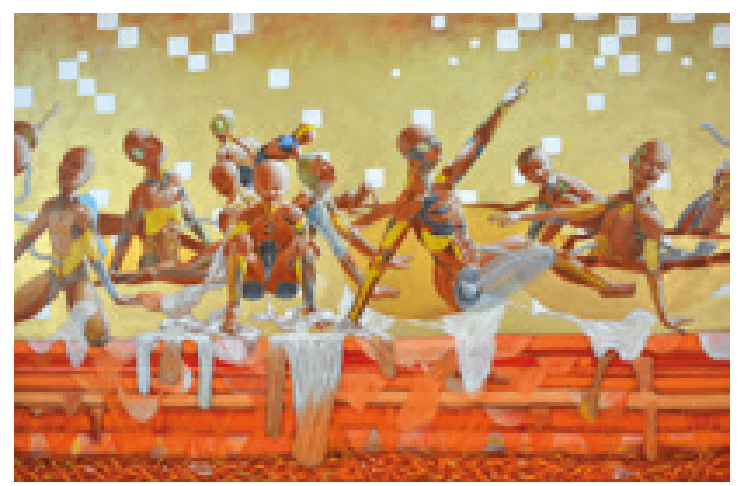

Figure 1. Bernard Paquet. L'an 3016, acrylique sur toile, 236 X 326 cm, 2016. Fonte: Bernard Paquet

1. http://www.idmais.org/pubs/PauloBernardino/2011/2011-4-3-PauloBB.pdf , consultée le 9 septembre 2016. 


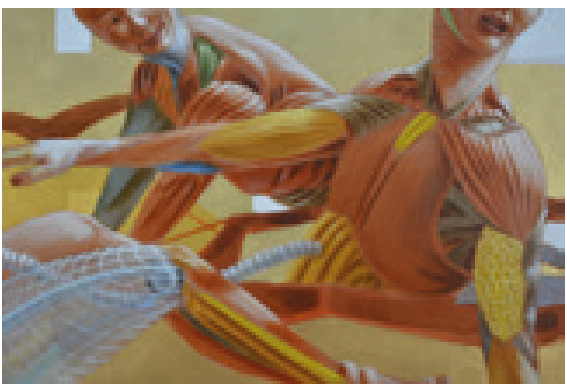

Figure 2. Bernard Paquet. L'an 3016, acrylique sur toile, 2016 (détail). Fonte: Bernard Paquet

Plus près de nous, les progrès de la biologie moderne, selon François Jacob ${ }^{2}$ font apparaître les êtres vivants "...constitués des mêmes modules qui sont distribués de manières différentes. Le monde vivant est une sorte de combinaison d'éléments en nombre fini..." qui auraient été soumis à un bricolage de l'évolution. De telle sorte que la médecine a pu considérer le corps comme un assemblage de morceaux manipulables qu'il est aisé de substituer par des greffes en cas de défaillance. Et maintenant, à l'ère du cyborg, les technologies et la science entretiennent l'espoir d'un corps augmenté, plus performant, voire immortel pour remplacer le corps de chair vu comme imparfait, dépassé, obsolète. Qui plus est, c'est la perspective de la performance, du rendement, de la vitesse, de la puissance et la communication qui semble dominer. S'impose alors un horizon dans lequel le corps ainsi modelé serait voué à ne devenir qu'un élément efficace de cybernétique ${ }^{3}$ pour assurer exclusivement une meilleure efficacité fonctionnaliste et économique dans un pur système de production et de communication. Bref, une machine augmentée pour le meilleur des mondes. Dans cette optique, la lucidité prémonitoire de Chaplin dans son film Les temps modernes laisse perplexe sur cette vision de rendre le corps plus productif.

II y a donc lieu de s'interroger, voire de s'inquiéter, à propos des conséquences de ce renouvellement du corps sur ce qu'on appelle la condition humaine. C'est pourquoi j'ai tenté de proposer ma propre projection face à cette problématique en mettant de l'avant une image du corps renouvelé non pas pour un meilleur rendement fonctionnaliste

2. François Jacob (La souris, la mouche et l'homme, Paris, Odile Jacob, 1997), in David Le Breton, Anthropologie du corps et modernité, Paris, Quadrige, 7ème éd. (Paris, PUF, 1990), p.324.

3. David Le Breton, op. cit. mais au contraire pour un bien-être amélioré et dans l'optique hédoniste d'un plaisir esthétique formel et chromatique. C'est le bricolage des formes et des couleurs en peinture qui le permet, contrairement à la pratique de d'autres artistes qui exploitent leurs corps comme médium.

\section{ET DES ARTISTES SUR LE CORPS}

Plusieurs artistes ont effectivement tenté d'exploiter les avancées technologiques pour opérer des transformations du corps avec le but d'expérimenter un humain amélioré et augmenté. On pense à l'artiste australien Stelarc qui déjà en 1981 voulait assurer une meilleure performance en dessin avec un troisième bras mécanique articulé comme un exosquelette qui suivait et reproduisait les gestes de son corps. Ou en 2015 lorsqu'il se fait implanter une oreille fonctionnelle dans l'avant-bras. Malgré ce type de prouesse, en dehors du moment de la performance artistique devant un public, il s'avère difficile pour un être humain de vivre et de se mouvoir en permanence avec un troisième bras qui est articulé. Pour sa part, l'artiste française Orlan a subi d'innombrables interventions chirurgicales dont plusieurs visaient à introduire des implants jusqu'à la limite du supportable du point de vue de la résistance du corps aux interventions chirurgicales. De son côté, en 1997, Eduardo Kac s'introduisit une puce dans la jambe pour s'inscrire dans la lancée de l'ajout de composantes électroniques dans la chair. Tout compte fait, voilà autant de performances à la fois artistiques et technologiques qui visent à démontrer que les capacités du corps peuvent être augmentées par les technosciences. Cependant, ces implants lui sont étrangers et peuvent représenter une menace pour son intégrité. Stelarc, par exemple, a dû être hospitalisé suite à des infections qui ont failli lui coûter son bras ${ }^{4}$ et admet que son projet de sculpture se développant dans l'estomac en 1993 fut très dangereux ${ }^{5}$.

Il est vrai que si le corps peut aujourd'hui carrément être exploité comme médium, c'est qu'il a maintenant atteint l'ère de sa reproductibilité technique ${ }^{6}$. Mais le problème de ces artistes est qu'ils veulent dépasser ce qui est la limite

4.http://www.ledevoir.com/societe/science-et-technologie/309993/de-I-humainau-robot-sapiens, consultée le 19 septembre 2016.

5.http://www.ledevoir.com/societe/science-et-technologie/309988/trois-bras-deuxjambes-et-une-sculpture-dans-I-estomac, consultée le 19 septembre 2016.

6. David Le Breton, op. cit., p.373. 
même de leurs actions, c'est-à-dire leur propre corps. Au contraire, d'autres artistes comme Aziz et Cucher agissent en quelque sorte par procuration car ils utilisent la photographie pour gommer les visages ou les parties génitales, ce qui est évidemment sans danger pour la personne et sans limite pour l'imagination. II en va de même en sculpture pour une partie de la production de la coréenne Lee Bull. C'est en ce sens que je considère qu'il m'est plus aisé d'aborder la question du posthumain par la fiction picturale et le multiple de la peinture.

\section{UNE IMAGE DU CORPS MULTIPLIÉE PAR LA PEINTURE}

Pour multiplier le corps, je considère deux paramètres : des éléments anatomiques qui se prêtent facilement au multiple et à des variations, puis l'absence de dualité.

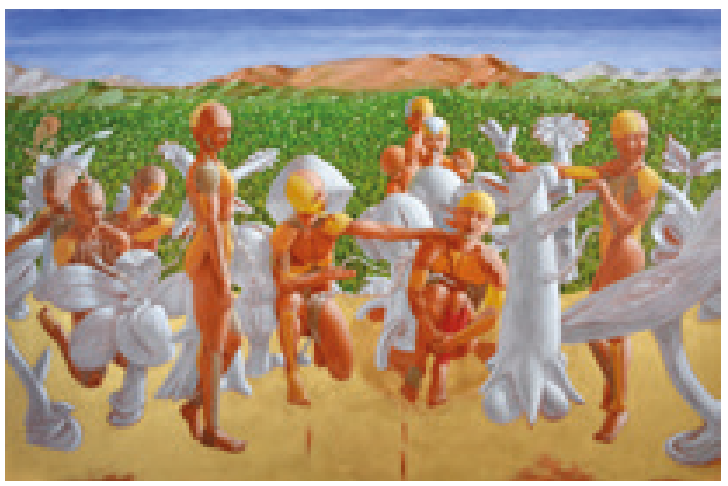

Figure 3. Bernard Paquet. L'an 3015, acrylique sur toile, 236 X 326 cm, 2015. Fonte: Bernard Paquet

Le multiple du corps du futur est souligné par Stelarc lorsqu'il déclare que le design biologique du corps pourrait "...être remodelé afin de faciliter le remplacement des organes endommagés ${ }^{7}$. Dans cette optique de substitution et de régénérescence, j'utilise la musculation et l'ossature afin de jouer avec des éléments qui se prêtent à la multiplication des modifications de formes et de couleurs dans une sorte de bricolage organisé. D'une part, chaque élément anatomique est conçu comme distinct, ce qui me permet de le traiter pour lui-même, librement, au niveau de la couleur. D'autre part, il demeure solidaire des autres éléments, pour deux raisons. La première est qu'il doit

7.http://www.revue-quasimodo.org/PDFs/7\%20-\%20Cyborg\%20Utopie\%20 Technologie.pdf, p.238, consultée le 3 mars 2015. s'insérer dans une structure générale qui permet de construire un corps qui demeure familier à notre regard; la seconde est sa participation à la composition purement picturale au niveau des formes et des couleurs. Cela me permet d'envisager une infinité de combinaisons et de permutations sans tenir compte de la réalité des couleurs de la chair et ce, en l'absence de l'épiderme. Le muscle deltoïde par exemple peut varier de forme et aussi bien être jaunâtre, rougeâtre, brunâtre ou verdâtre puisque c'est la peinture, et non une certaine réalité externe, qui dicte ses lois (Figure 2). J'utilise une gamme qui se base sur cinq couleurs (ocre jaune, Terre de Sienne naturelle, Terres de Sienne brûlée, Terre d'ombre naturelle et Terre d'ombre brûlée), ce qui donne une certaine cohérence aux morphologies ainsi qu'à la peinture sous l'angle de l'équilibre d'une composition et de l'accord entre les couleurs (Figure 5). Ainsi, par des choix chromatiques différents pour l'édification de chaque corps, j'exploite le multiple ; l'image du corps se répète mais se présente à chaque fois modifiée dans ses variations. Aux couleurs s'ajoute la texture des surfaces, soit striées ou lisses, à l'image des deux catégories de muscles qui existent. Les éléments morphologiques peints conservent cependant chacun leur singularité parce que les stries sont à chaque fois traitées différemment, que ce soit dans leur nombre, leur largeur ou leur profondeur. La multiplication des corps se fait donc dans cette différence de formes, de couleurs et de textures et c'est cette multiplication dans la différence qui construit et solidifie le concept imagé du posthumain.

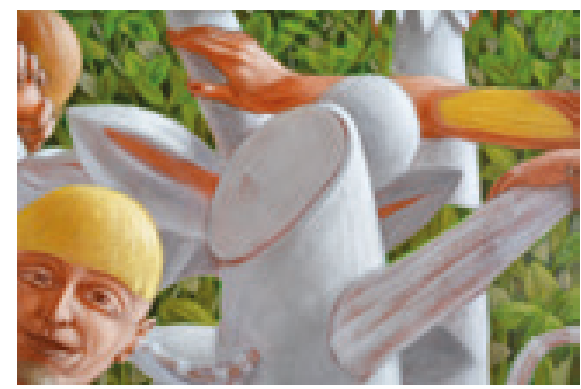

Figure 4. Bernard Paquet. L'an 3015, acrylique sur toile, 2015 (détail). Fonte: Bernard Paquet

Encore pour assurer une cohérence, j'ai voulu jouer avec un seul type de combinaison plutôt que de distinguer deux classes de corps via la dualité des deux genres que sont le masculin et le féminin. Et cela selon deux aspects. Le premier 
relève de l'histoire ancienne; c'est une manière de s'inscrire dans le fantasme de l'unité originelle qui remonte au mythe grec tel qu'il est relaté dans le Symposium de Platon à propos d'Éros et du troisième sexe (mâle femelle) ${ }^{8}$. Celui-ci, réunissant les forces des deux sexes, est considéré trop puissant par Zeus qui le fracture par un éclair en deux parties mâle et femelle) qui par la suite cherchent à se réunifier. Mais dans une autre version du Symposium, Éros est plutôt présenté comme une diaspora d'un corps en fragments flottants, dont la préoccupation première est la recherche des pièces corporelles en vue de se dupliquer plutôt que la quête de l'autre dans l'espoir d'une fusion des différences. C'est donc en ce second sens que je joue avec des fragments anatomiques afin de les assembler, mais d'une manière à chaque fois différente dans les formes, les textures et les couleurs, tout en demeurant dans un type de construction qui ressemble au corps humain. Éros serait ainsi toujours présent, mais en tant que troisième sexe.

Le second aspect est celui d'un monde sans genre, tel que conçu par Donna Haraway qui dans son célèbre manifeste Cyborg Manifesto ${ }^{9}$, entend éliminer les dualités qui opposent la femme à l'homme, le corps à l'esprit, la nature à la culture, la naturel à l'artificiel. Son concept de cyborg est avant tout féministe et socialiste; il n'est plus pensé pour accroître les performances selon un modèle masculin de virilité qui, selon elle, caractérise le cyborg, mais il doit au contraire s'ouvrir à la multiplicité et à l'indétermination, annulant ainsi les conflits entre le masculin et le féminin. Pour elle, " être autre, c'est être multiple ${ }^{10}$, sans se préoccuper de quelconques oppositions qui, en réalité, entraînent des divisions.

\section{DES MONDES MULTIPLES ET MULTIPLIÉS POUR UNE AURA}

Dès lors, l'autre posthumain que je peins est toujours conçu comme un "nouvel autre», libéré des oppositions duelles et constamment renouvelé au travers de sa propre multiplication. Et lorsque j'assemble ces multiples

8.http://www.e-flux.com/architecture/superhumanity/68674/masters-and-slaves/, consultée le 19 octobre 2016.

9. Donna Haraway, Simians, Cyborgs and Women : The reinvention of Nature, New York, Routledge, 1991.

10.http://www.revue-quasimodo.org/PDFs/7\%20-\%20Cyborg\%20Utopie\%20 Technologie.pdf, p.238, consultée le 3 mars 2015. posthumains sans genre dans des compositions, je propose par le fait même des mondes. Pour le philosophe américain Nelson Goodman, il n'y a pas un monde, mais des mondes qui se fabriquent et il existe plusieurs manières d'en fabriquer par les constructions, surtout chez les artistes et les scientifiques. Mais on "...démarre toujours avec des mondes déjà à disposition : faire, c'est refaire...." ${ }^{11}$. Par conséquent, chaque monde nouveau peint s'inscrit dans la génération des multiples qui l'ont précédé. En ce sens, je propose des mondes inédits mais à partir de référents qu'on peut identifier à la nature ou à des moyens picturaux historiques qui se sont répétés au travers des siècles : ligne d'horizon, ciel bleu, végétation, fond doré sans ombre et intemporel, sous-sol de strates, etc... Ces représentations s'appuient sur un paradoxe visuel qui est répété. II met en relation l'illusion d'une profondeur naturaliste (le paysage, le sous-sol) et l'à-plat doré dans lequel l'absence d'ombres portées introduit une intemporalité et un non-lieu, voire un rappel du sacré de la peinture ancienne.

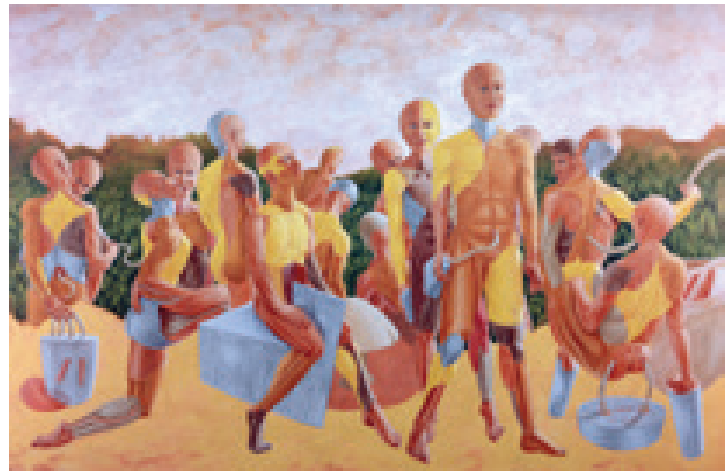

Figure 5. Bernard Paquet. L'an 3014, acrylique sur toile, 236 X 326 cm, 2014. Fonte: Bernard Paquet

Travailler à faire des multiples à partir d'une invention picturale initiale et de moyens historiques ajoute cependant un aspect fondamental au processus de multiplication qui contribue à édifier un monde. Lors d'une conférence à laquelle j'assistais à la Sorbonne, le peintre français Henri Cueco montra une série d'images de ses toiles représentant des patates. II expliqua qu'il n'y avait pas une façon de créer une patate en peinture (la patate objective), mais bien une infinité de manières de telle sorte que le

11. http://www.seroux.be/spip.php?article181, consultée le 30 octobre 2016. 
nombre de représentations toutes singulières contribuait à inventer, à consolider et à élargir le « monde de ce légume », autrement dit l'idée qu'on s'en fait. Et, ajouta-t-il, la série s'accroissait simplement parce qu'il essayait à chaque nouvelle toile de trouver une autre manière d'inventer l'image de la pomme de terre. C'est ainsi qu'un monde et des corps naissent en peinture et prennent de l'expansion en se précisant de plus en plus.

Cela n'est pas sans nous rappeler la répétition chez Deleuze ${ }^{12}$ qui montre que la répétition du «même» dépasse la simple addition pour créer une nouvelle identité. Dans ma peinture, le même est entendu comme une catégorie, soit celle du corps humain identifiable à un écorché multicolore. Cependant, la stratégie picturale consiste à miser sur des variations de couleurs, de formes, de proportions, de postures et de composition qui agrandissent le spectre de représentation du corps posthumain. Autrement dit, ce type de corps n'est véritablement identifiable que par le nombre croissant de ses diverses variations.

On peut illustrer ce processus par l'exemple de la forêt chez Francis Ponges. Pour ce dernier, une forêt est composée d'une multitude de feuilles, d'arbres, d'arbustes et de plantes diverses ${ }^{13}$ au centre desquels se trouve le cœur de cette forêt, c'est-à-dire son identité. II ajoute que si on enlève ces éléments, le cœur disparaît. Cela signifie, en ce qui me concerne, qu'il n'y a pas, à l'instar de la patate, une présentation objective du posthumain (un référent qui existe) mais qu'il se crée une identité par le processus de multiplication de la peinture qui dessine le cœur de cette identité au fur et à mesure de l'accumulation des multiples de ce corps inséré dans des mondes. II s'agit plus précisément de multiples d'anatomies colorées éclairées sans ombre portée, de multiples de fond doré pour marquer l'intemporel et l'irréel, de multiples de divisions horizontales pour donner une assise terrestre, etc.

Cette stratégie picturale du multiple favorise une expansion qui est en relation directe avec l'aura, telle qu'on la connait chez Walter Benjamin. On sait que, selon lui, l'ère de la reproductibilité technique a fait disparaître l'aura liée à l'unicité d'un phénomène ou d'une œuvre d'art. Toutefois,

12. Gilles Deleuze, Différence et répétition, Paris, PUF, 1968.

13. Francis Ponges, Méthodes, Paris, Gallimard, 1988. il nous indique dans le même texte célèbre ${ }^{14}$ que le fait de multiplier les reproductions d'une chose est l'indice d'un événement importance et fait naître une nouvelle aura. Le meilleur exemple est celui de la Joconde dont les innombrables reproductions, pastiches et commentaires ont créé une immense aura, au lieu de la diminuer. C'est pourquoi en peinture la pratique de la sérialité par le multiple des variations du même (i.e. la catégorie corps écorché multicolore et celle de la combinaison fond doré et ligne d'horizon) permet de créer un monde, d'en assurer le cœur et d'espérer une aura. Si le premier corps posthumain que j'ai peint était un écorché étrange et animé, même pour moi, il est devenu par la suite un multiple parmi les autres et l'image de l'écorché coloré a pris graduellement tout son sens et son rayonnement au fur et à mesure de ses multiples variations, donnant toute la légitimité à un corpus qui, ainsi, devient familier et s'installe dans le monde.

\section{L'HÉRITAGE DE L'ÉCORCHÉ}

Les variations de la catégorie " écorché " répondent évidemment à une tradition qui remonte à la Renaissance. Dans son récent ouvrage sur les études anatomiques du XVlème au XVIllème siècle ${ }^{15}$, Raphael Cuir distingue deux approches. La seconde, qui est familière à notre esprit scientifique, expose le corps comme un mécanisme objectif qui fait abstraction de la vie : c'est à l'origine le corps-machine de Descartes. Le sujet est ainsi évacué d'un organisme qui est réduit à un pur objet d'étude scientifique dont la représentation est toujours figée dans une perspective purement clinique, c'est-à-dire inanimée. Au contraire, la première approche, typique de la Renaissance, lie le sujet au corps, comme si un écorché conservait une certaine âme. Elle est illustrée par les études anatomiques d'André Vésale (De humani corporis fabrica, 1543) et de Charles Estienne (De dissectione partium corporis humani, 1539-1545) qui ont la particularité de contenir des figures animées. Celles-ci relèvent, selon la thèse de Cuir, "...à la fois de la fragilité du corps humain et de la puissance du Créateur...»"16 dans un esprit humaniste qui teinte les représentations d'écorchés

14. Walter Benjamin, L'CEuvre d'art à l'époque de sa reproductibilité technique (1939), traduit par Frédéric Joly, préface d'Antoine de Baecque, Paris, Payot, coll. Petite Bibliothèque Payot, 2013.

15. Raphael Cuir, Renaissance de l'anatomie, Paris, Hermann, 2016

16. Yves Hersant, Avant-propos, in Raphael Cuir, Renaissance de l'anatomie, Paris, Hermann, 2016, p.9. 
d'une attitude humaine très subjective jusqu'à installer une dimension érotique dans les interprétations d'Estienne, par exemple. Pour Cuir, ce sont «...des figures discursives qui ne parlent pas seulement d'anatomie, mais aussi d'Éros et de Thanatos.... ${ }^{17}$ et qui ainsi comportent "...une pratique réflexive, un savoir qui se donne dans la relation à soi en tant que sujet vivant ${ }^{18}$, répondant à la célèbre maxime " Connais-toi toi-même ", gravée sur le fronton du temple de Delphes. Cela perdure même à l'époque de Descartes alors que certaines images, comme celles de Jacques Gautier d'Agoty, affichent encore des anatomies prenant des positions du vivant dont seuls le tronc, les jambes et les bras sont écorchés, alors que les têtes qui nous regardent sont conservées avec toute leur expression épidermique.

C'est dans cette perspective subjective que j'inscris ma conception de l'image du corps posthumain qui exploite l'idée de l'écorché en y ajoutant une forte dimension subjective afin de suggérer fortement la vie, en me jouant des exactitudes anatomiques. Et, comme chez Gautier d'Agoty, les têtes sont souvent moins écorchées que les corps. Par la combinaison de cette volonté avec l'utilisation de mes connaissances anatomiques et de certaines ressources d'illustration médicale ${ }^{19}$, je reprends, comme Stelarc, "l'idée du corps comme architecture évolutive ${ }^{20}$, sans toutefois proposer comme ce dernier l'exploration d'une structure anatomique alternative. Certes, le corps posthumain demeure un corps dans sa structure générale, mais, outre la fantaisie des couleurs et des textures, j'y insère des fantaisies musculaires, quelques éléments non organiques et des formes dont la réalité relève plus de la seule peinture que d'une réalité externe, car la peinture prime sur le corps.

Le corps posthumain est donc un assemblage de pièces anatomiques qui prend sa liberté face à l'exactitude morphologique et à l'histoire des représentations picturales. Je le multiplie afin d'installer une post-humanité qui donne une légitimité à la représentation, de la même manière qu'il est nécessaire d'avoir des milliards d'humains pour parler

17. Raphael Cuir, op. cit., p.32.

18. Loc. cit.

19. Jean-Marc Bourgery et Nicolas-Henri Jacob, Atlas d'anatomie humaine et de chirurgie, sl, Taschen, 2012

20. Marie Lechner, "Le corps amplifié de Stelarc», in Libération, édition électronique du 12 octobre 2007, http://next.liberation.fr/culture-next/2007/10/12/ le-corps-amplifie-de-stelarc_103649, consulté le 21 janvier 2017. d'une humanité. Dès lors, l'image d'une post-humanité se développe et se précise à la fois par la construction de chaque corps et par la cohésion avec le multiple des corps déjà peints et de ceux à venir, à la fois par le choix de la gamme chromatique et par sa répétition, ouverte et infinie. Cela s'inscrit à l'intérieur de la peinture elle-même qui se répète ; la répartition des multiples d'une couleur ou d'une forme est d'abord une affaire de composition, c'est-à-dire de distribution des multiples, ainsi que la multiplication des superpositions de couches visant la solidité chromatique. C'est ainsi que le processus de la peinture produit du multiple et que le multiple est nécessaire pour donner du corps à la peinture.

\section{RÉFÉRENCES}

AMADO, Mónica Alexandra Fernandes Marques e BASTOS, Paulo Bernardino das Neves. Performatividade do corpo pós-humano: de Narciso a Pigmalião. 2011. Disponible à: http://www.idmais.org/pubs/PauloBernardino/2011/2011-4-3PauloBB.pdf, consultée le 9 septembre 2016.

BENJAMIN, Walter. L'CEuvre d'art à l'époque de sa reproductibilité technique (1939), traduit par Frédéric Joly, préface d'Antoine de Baecque. Paris: Payot, coll. Petite Bibliothèque Payot, 2013.

BOURGERY, Jean-Marc et JACOB, Nicolas-Henri. Atlas d'anatomie humaine et de chirurgie. sl. Cologne: Taschen, 2012.

CUIR, Raphael. Renaissance de l'anatomie. Paris: Hermann, 2016.

DELEUZE, Gilles. Différence et répétition. Paris: PUF, 1968.

GOODMAN, Nelson. La fabrique des mondes. Disponible à: http:// www.seroux.be/spip.php?article181, consultée le 30 octobre 2016.

GRUGIER, Maxence. L'utopie cyborg: Réinvention de l'humain dans un futur sur-technologique. 2003. Disponible à: http:// www.revue-quasimodo.org/PDFs/7\%20-\%20Cyborg\%20 Utopie\%20Technologie.pdf, p.238, consultée le 3 mars 2015.

HARAWAY, Donna. Simians, Cyborgs and Women: The reinvention of Nature, New York : Routledge, 1991.

LE BRETON, David. Anthropologie du corps et modernité. Quadrige, 7ème éd. Paris : PUF, 1990.

LECHNER, Marie. Le corps amplifié de Stelarc. In: Libération, édition électronique du 12 octobre 2007, http://next. liberation.fr/culture-next/2007/10/12/le-corps-amplifie-destelarc_103649, consulté le 21 janvier 2017. 
LE DEVOIR. De I'humain au Robot sapiens. 2010. Disponible à: http://www.ledevoir.com/societe/science-ettechnologie/309993/de-I-humain-au-robot-sapiens, consultée le 19 septembre 2016.

LE DEVOIR. Trois bras, deux jambes et une sculpture dans I'estomac. 2010. Disponible à: http://www.ledevoir.com/ societe/science-et-technologie/309988/trois-bras-deuxjambes-et-une-sculpture-dans---estomac, consultée le 19 septembre 2016.

KALLIPOLITI, Lydia. Masters and Slaves. Disponible à: http:// www.e-flux.com/architecture/superhumanity/68674/mastersand-slaves/, consultée le 19 octobre 2016.

PONGES, Francis. Méthodes. Paris: Gallimard, 1988.

Bernard Paquet: Professeur titulaire à l'École des Arts Visuels de I'Université Laval, Québec, Canada. Docteur en Arts et Sciences de I'Art à I'Université de Paris I, Panthéon-Sorbonne (1997). II a tenu de nombreuses expositions individuelles et collectives au Canada, en France, au Brésil, à Monaco et en Tunisie. Il a publié des articles dans de nombreux magazines ou livres et donné des conférences sur le processus créatif au Brésil, au Canada, en France, en Angleterre, en Martinique, à Monaco, en Suisse et en Tunisie.

(*) Interview envoyée en agosto 2017. 